\title{
Effect of Laser Irradiation on the Properties of ZnO Buffer Layers and Its Application to Selective-Growth of ZnO Nano/Microcrystals
}

\author{
Tetsuya SHIMOGAKI ${ }^{* 1}$, Hirotaka KAWAHARA ${ }^{* 1}$, Shihomi NAKAO ${ }^{* 1}$, Kosuke HARADA*1 ${ }^{\text {, Mitsuhiro HIGASHIHATA }}{ }^{* 1}$ \\ Hiroshi IKENOUE $^{* 1}$, Yoshiki NAKARA ${ }^{* 2}$, Daisuke NAKAMURA ${ }^{* 1}$, Tatsuo OKADA ${ }^{* 1}$ \\ ${ }^{*}$ Graduate School of Information Science and Electrical Engineering, Kyushu Univ., 744 Motooka, \\ Nishi-ku, Fukuoka 819-0395, Japan \\ E-mail: shimogaki@laserlab.ees.kyushu-u.ac.jp \\ ${ }^{* 2}$ Faculty of Information Science and Electrical Engineering, Kyushu Univ., 744 Motooka, Nishi- \\ ku, Fukuoka 819-0395, Japan
}

\begin{abstract}
$\mathrm{ZnO}$ nanocrystals, which are characterized by their configurations and fine structures, are unique oxide semiconductors. They are synthesized by nanoparticle-assisted pulsed laser deposition on $\mathrm{ZnO}$ buffer layers deposited on $a$-cut sapphire substrates. In this report, the new possibilities of $\mathrm{UV}$ laser-processing to the field of $\mathrm{ZnO}$ buffer layers and $\mathrm{ZnO}$ nano/microcrystals are suggested. Effects of ultraviolet laser-irradiation on morphological and electrical properties of $\mathrm{ZnO}$ buffer layers were investigated. After laser irradiation, surface work function increased in the region laserirradiated at $300 \mathrm{~mJ} / \mathrm{cm}^{2}$, whereas it decreased in the region laser-irradiated at $500 \mathrm{~mJ} / \mathrm{cm}^{2}$. Then, $\mathrm{ZnO}$ nanowires were synthesized on the $\mathrm{ZnO}$ buffer layers locally laser-irradiated at $300 \mathrm{~mJ} / \mathrm{cm}^{2}$ and $500 \mathrm{~mJ} / \mathrm{cm}^{2}$ by nanoparticle-assisted pulsed laser deposition. Number densities of $\mathrm{ZnO}$ nanowires were varied depend on laser fluences. It decreased in the region pre-laser-irradiated at 300 $\mathrm{mJ} / \mathrm{cm}^{2}$, whereas it increased in the region pre-laser-irradiated at $500 \mathrm{~mJ} / \mathrm{cm}^{2}$. Additionally, it was demonstrated that periodically-aligned $\mathrm{ZnO}$ microcrystals can be synthesized using four-beam interference laser irradiation to the $\mathrm{ZnO}$ buffer layers followed by nanoparticle-assisted pulsed laser deposition. Since these processes do not require any catalysts, they are expected to be the new fabrication method for $\mathrm{ZnO}$ nano/micro crystals.
\end{abstract}

DOI: $10.2961 / \mathrm{jlmn} .2015 .03 .0004$

Keywords: zinc oxide, nanowire, microcrystal, laser annealing, laser ablation, four-beam interfered laser

\section{Introduction}

Zinc oxide $(\mathrm{ZnO})$, which has abundant natural resources, has a wide band-gap energy of approximately 3.37 $\mathrm{eV}$ and a large exciton binding energy of $60 \mathrm{meV}$. These mean that $\mathrm{ZnO}$ can realize an efficient exciton emission in near ultraviolet (UV) spectral region. Accordingly, $\mathrm{ZnO}$ is one of the most attracted materials for optoelectronic applications which operate in the UV range. $[1,2]$ In addition, a variety of $\mathrm{ZnO}$ nanocrystals can be synthesized due to selforganization. Besides, a variety of $\mathrm{ZnO}$ nanocrystals such as nanowires, nanorods, and nanorings, which are provided by their self-organized growth, have scientific attractiveness because of their unique morphologies. [3, 4, 5] $\mathrm{ZnO}$ nanocrystals have quite-small volume and high aspect ratio, so that quantum effect and optical confinement can be expected.

In this paper, we suggest new ideas about the combination of the $\mathrm{ZnO}$ crystals and UV laser processing. There are some associated researches. Pulsed-laser deposition (PLD) is a well-known method of fabricating $\mathrm{ZnO}$ thin films characterized by a high-throughput. [6, 7] In our early study, nanoparticle-assisted pulsed laser deposition (NAPLD) was established to fabricate $\mathrm{ZnO}$ nanocrystals. [8] Additionally, it was found that vertical-growth-degree of $\mathrm{ZnO}$ nanowires can be improved using $\mathrm{ZnO}$ buffer layer. [9] UV laser annealing on $\mathrm{ZnO}$ crystals have been investigated by some researchers. For example, the effect of laser annealing on $\mathrm{ZnO}$ thin film was researched and it was found that the surface roughness of $\mathrm{ZnO}$ thin films can be decreased. [10] Optical properties of $\mathrm{ZnO}$ nanocrystals have been investigated in our previous study. [11]

In this paper, a new idea about the combination of PLD, laser annealing and NAPLD is suggested. Flow chart of experimental procedures were shown in Figure 1. At first, a $\mathrm{ZnO}$ buffer layer, which is a kind of $\mathrm{ZnO}$ thin films, was laser-annealed at some fluences, then $\mathrm{ZnO}$ nanowires were grown on it. The variation in the number density of $\mathrm{ZnO}$ nanowires was investigated. Subsequently, periodicallyaligned $\mathrm{ZnO}$ microcrystals were fabricated using the micropatterned $\mathrm{ZnO}$ buffer layer by irradiating four-beam interfered the third harmonic of Nd:YAG laser.

\section{Experiment}

\subsection{Preparation of $\mathrm{ZnO}$ buffer layers}




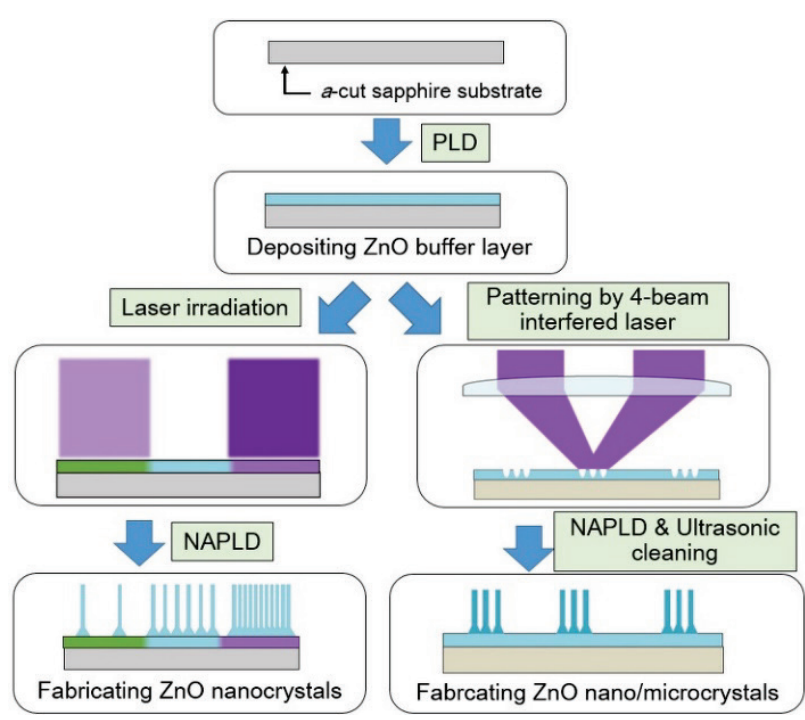

Fig. 1 Schematic flow chart of experimental procedures.

$\mathrm{ZnO}$ thin films, which are called $\mathrm{ZnO}$ buffer layers hereafter, were synthesized by PLD. A sintered $\mathrm{ZnO}$ target placed in a vacuum chamber was ablated for 5 min with the third harmonic of a Q-switched Nd:YAG laser $(\lambda=355$ nm, pulse width $\tau=10 \mathrm{ns,} \mathrm{Quanta-ray} \mathrm{GCR-290-10,} \mathrm{Spec-}$ tra-Physics) at a fluence of $1.5 \mathrm{~J} / \mathrm{cm}^{2}$. The chamber pressure were kept at $3 \mathrm{~Pa}$. $\mathrm{ZnO}$ buffer layers were deposited on an $a$-cut sapphire substrate heated at $500{ }^{\circ} \mathrm{C}$, placed at the facing position toward the sintered $\mathrm{ZnO}$ target.

\subsection{Pre-laser annealing and pre-laser ablation}

Pre-laser irradiating process was performed with a $\mathrm{KrF}$ excimer laser $(\lambda=248 \mathrm{~nm}$, pulse width $\tau=55 \mathrm{~ns})$. The laser beam was scanned over the surface of the $\mathrm{ZnO}$ buffer layer using a motorized $\mathrm{X}-\mathrm{Y}$ stage. The repetition rate and scanning speed were set at $1000 \mathrm{~Hz}$ and $3.5 \mathrm{~mm} / \mathrm{s}$, which the $\mathrm{ZnO}$ buffer layer was irradiated 100 times per location. Laser fluences were $300 \mathrm{~mJ} / \mathrm{cm}^{2}$ and $500 \mathrm{~mJ} / \mathrm{cm}^{2}$, as shown in Fig. 2(a).

Figure 2(b) and (c) show atomic force microscopy (AFM) and kelvin probe force microscopy (KPFM) images of the boundary between the laser-irradiated region at 300 $\mathrm{mJ} / \mathrm{cm}^{2}$ and as-deposited region. Both images were obtained concurrently, so that the observing areas are same. It was found that the root mean square value of the surface RMS roughness (Root-Mean-Square value) of the $\mathrm{ZnO}$ buffer layer decreased from $3.136 \mathrm{~nm}$ to $0.548 \mathrm{~nm}$ by laser irradiating at $300 \mathrm{~mJ} / \mathrm{cm}^{2}$. The KPFM image in Fig. 2(c) indicates the increase in the surface work function of $\mathrm{ZnO}$ buffer layer by laser irradiation at $300 \mathrm{~mJ} / \mathrm{cm}^{2}$, because the mapping parameter of KPFM image, which is the contact potential difference between the cantilever and the surface of the sample $\left(V_{C P D}\right)$, is represented by the following equation. [12]

$$
e V_{C P D}=\Phi_{\text {probe }}-\Phi_{\text {sample }}
$$

Here, $\Phi_{\text {probe }}$ and $\Phi_{\text {sample }}$ are the work functions of the cantilever and sample, respectively, and $e$ is the elementary charge. Therefore, the contrast of KPFM images provides the information of inversed-contrast of surface work function of the sample.

Figure 2(d) and (e) show the AFM and KPFM images of the boundary between the as-deposited region and laserirradiated region at $500 \mathrm{~mJ} / \mathrm{cm}^{2}$. There are an energy leak and interference of the laser beam, so that as-deposited region have blue area. Additionally, since the region laserirradiated at a fluence of $500 \mathrm{~mJ} / \mathrm{cm}^{2}$ was chipped off due to excess laser fluence, the average surface height decreased by laser irradiation at a fluence of $500 \mathrm{~mJ} / \mathrm{cm}^{2}$. The RMS roughness decreased from $3.451 \mathrm{~nm}$ to $0.699 \mathrm{~nm}$. Since KPFM contrast is often affected by precipitous variation in surface height, the probe detects the potential with a slight mechanical error at around the boundary in the bottom of half of Fig. 2(e). The KPFM image indicates that the surface work function of $\mathrm{ZnO}$ buffer layer was decreased by laser irradiation at a fluence of $500 \mathrm{~mJ} / \mathrm{cm}^{2}$.

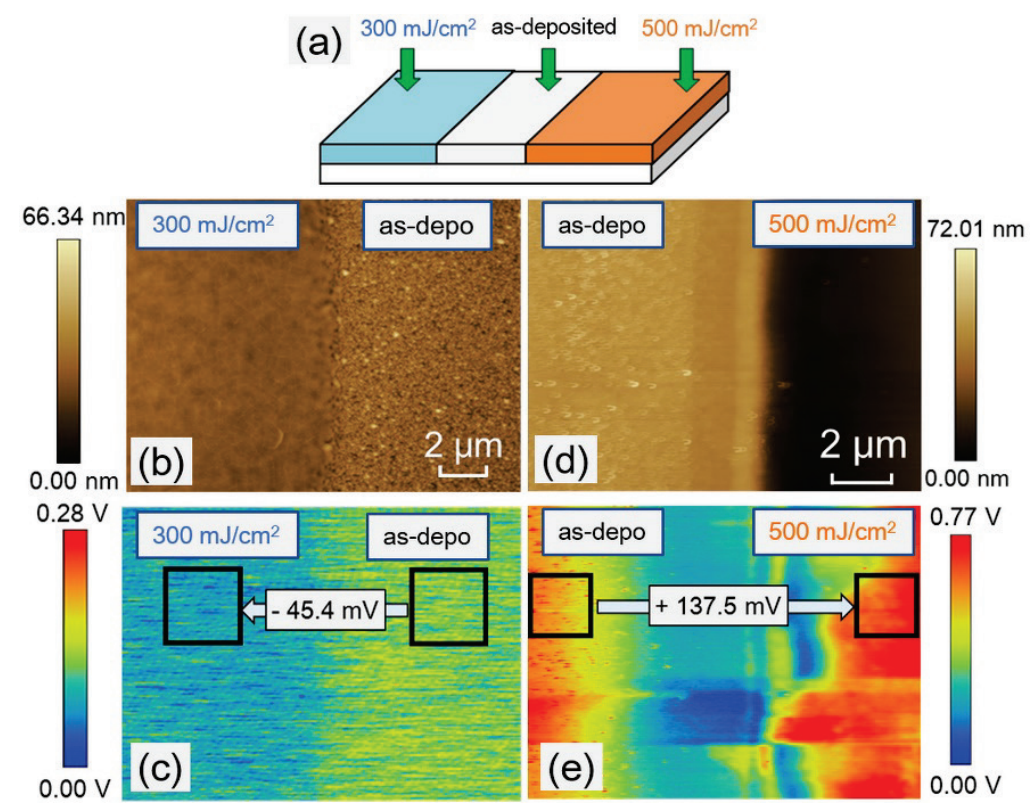

Fig. 2 (a) Schematic of the laser-irradiated $\mathrm{ZnO}$ buffer layer. (b) AFM and (c) KPFM images of the laser-irradiated ZnO buffer layer at the boundary between the laser-irradiated region at $300 \mathrm{~mJ} / \mathrm{cm}^{2}$ and as-deposited region. (d) AFM and (e) KPFM images of it at the boundary between the as-deposited region and laser-irradiated region at $500 \mathrm{~mJ} / \mathrm{cm}^{2}$. 


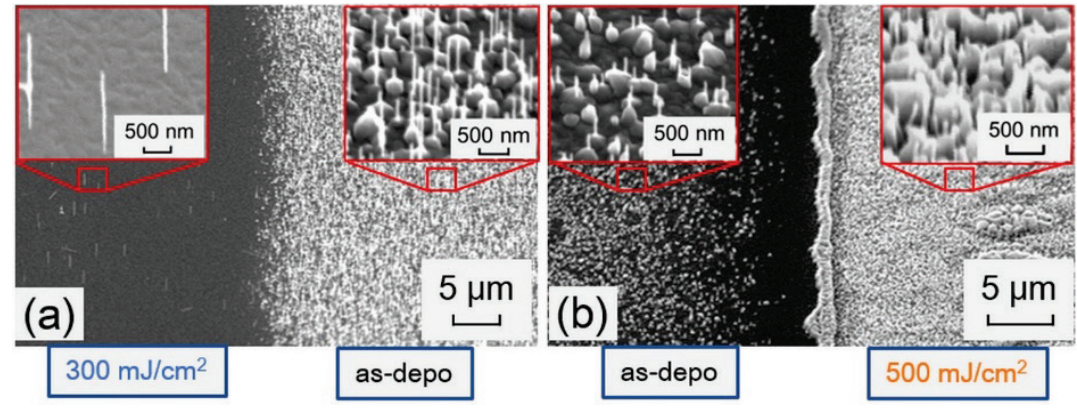

Fig. 3 Tilted SEM images of $\mathrm{ZnO}$ nanowires grown on $\mathrm{ZnO}$ buffer layer. (a) At the boundary between pre-laser irradiated region at $300 \mathrm{~mJ} / \mathrm{cm} 2$ and non-irradiated region and (b) at the boundary between non-irradiated region and pre-laser irradiated region at 500 $\mathrm{mJ} / \mathrm{cm} 2$.

The variations in properties of $\mathrm{ZnO}$ buffer layers can be explained by effects of the laser annealing and laser ablation. Laser irradiation at appropriate fluence can be regarded as a laser annealing process. On the other hand, laser irradiation at excess fluence can be regarded as a laser ablation process. We believe that the decrease in the surface work function of $\mathrm{ZnO}$ buffer layers was caused by crystalline defects induced by laser ablation. Additionally, one of the reasons for the increase of work function by laser annealing was the decrease of crystal grain boundaries, where various crystalline defects concentrate. In $\mathrm{ZnO}$ crystals, the most susceptible defects are oxygen vacancies and interstitial zincs. [13] Because both defects lead $n$-type conductions, the Fermi level decreased at the laser-annealed region. Accordingly, work functions were increased and decreased at laser-annealed region and laser-ablated region, respectively.

\subsection{Fabrication of $\mathrm{ZnO}$ nanowires on the laser- irradiated $\mathrm{ZnO}$ buffer layer}

Vertically-aligned $\mathrm{ZnO}$ nanowires were fabricated on the pre-laser irradiated $\mathrm{ZnO}$ buffer layer, using NAPLD. In this process, $\mathrm{ZnO}$ sintered target was ablated for $20 \mathrm{~min}$ by the third harmonic of a Q-switched the third harmonic of $\mathrm{Nd}$ :YAG laser at the same laser parameters and experimental geometry as PLD. The chamber was filled with Ar gas at a pressure of $26700 \mathrm{~Pa}$. $\mathrm{ZnO}$ nanoparticles, which are precursor materials of $\mathrm{ZnO}$ nanowires, were generated due to the relatively high pressure. $\mathrm{ZnO}$ nanoparticles were deposited on pre-laser irradiated $\mathrm{ZnO}$ buffer layer heated at $750{ }^{\circ} \mathrm{C}$.

Figures 3(a) and (b) show the images of $\mathrm{ZnO}$ nanowires observed by a scanning electron microscopy (SEM, VE7800, KEYENCE). The left side of Fig. 3(a) is the pre-laser annealed region at $300 \mathrm{~mJ} / \mathrm{cm}^{2}$. It was found that the number density of $\mathrm{ZnO}$ nanowires decreased at the pre-laser annealed region at $300 \mathrm{~mJ} / \mathrm{cm}^{2}$. On the other hand, it increased at the right side of Fig. 3(b), which is the pre-laser ablated region at $500 \mathrm{~mJ} / \mathrm{cm}^{2}$. In both cases, $\mathrm{ZnO}$ nanowires were grown on the top of pyramidal crystals, as shown in insets of Fig. 3(a) and (b). The nucleation of pyramidal crystals generally took place at the early stage of NAPLD process.

It was resulted that pre-laser annealing at a moderate fluence decreases the nucleation rates of pyramidal core crystals and $\mathrm{ZnO}$ nanowires. On the other hand, pre-laser ablation at an excess fluence increases them. Accordingly,

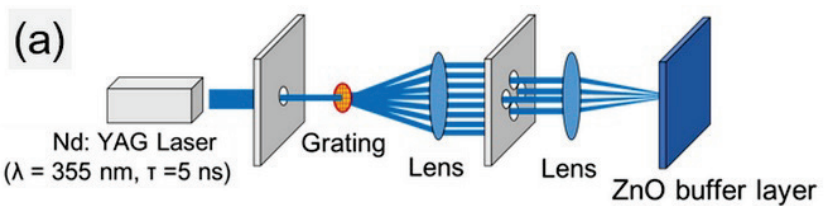

(b)

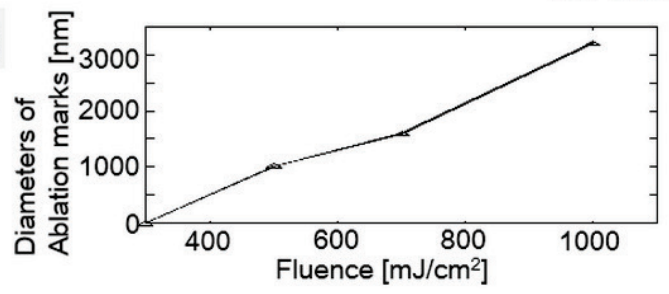

Fig. 4 (a) Schematic of four-beam laser interference irradiation system. (b) Dependence of diameters of ablation marks on the interference laser fluences.

KPFM images give projections of density of $\mathrm{ZnO}$ nanowires in advance of NAPLD process.

\subsection{Fabrication of $\mathrm{ZnO}$ microcrystals by micro pat- terning with four-beam interfered laser}

Next, periodically-aligned $\mathrm{ZnO}$ microwires were fabricated by micro-patterning on a $\mathrm{ZnO}$ buffer layer with fourbeam interference of the third harmonic of a Q-switched Nd:YAG laser (pulse width $\tau=5 \mathrm{~ns}$, Powerlite 8000, Continuum). Micro-patterning and fabrication of meta-material by four-beam interfered laser irradiation were reported by some researchers. [14] We applied it to make micro patterning on $\mathrm{ZnO}$ buffer layers followed by NAPLD. Figure 4(a) shows a schematic diagram of four-beam interference laser irradiation system. The incident laser was diffracted by the transmission grating, then diffracted lasers are collimated by a lens. Four first-order diffracted beams were extracted using a slit, then they were focused by another lens. Figure 4(b), where dependence of diameters of ablation marks on the interference laser fluences, indicates that the size of patterns can be controlled.

Figure 5(a) shows a SEM image of the micro-patterned $\mathrm{ZnO}$ buffer layer. It was found that ablation marks with diameters of less than $2.0 \mu \mathrm{m}$ were periodically-aligned on the $\mathrm{ZnO}$ buffer layer. They were fabricated on the area of $2.0 \times 10^{4} \mu \mathrm{m}^{2}$ by only the one pulse irradiation at a fluence of $700 \mathrm{~mJ} / \mathrm{cm}^{2}$. AFM and KPFM images, which are shown in Figs. 5(b) and (c), indicate that the laser-ablated region had lower surface work functions. According to the results as mentioned in section 2.2 and 2.3, densely-packed $\mathrm{ZnO}$ nanowires can be grown on laser-ablated region. 


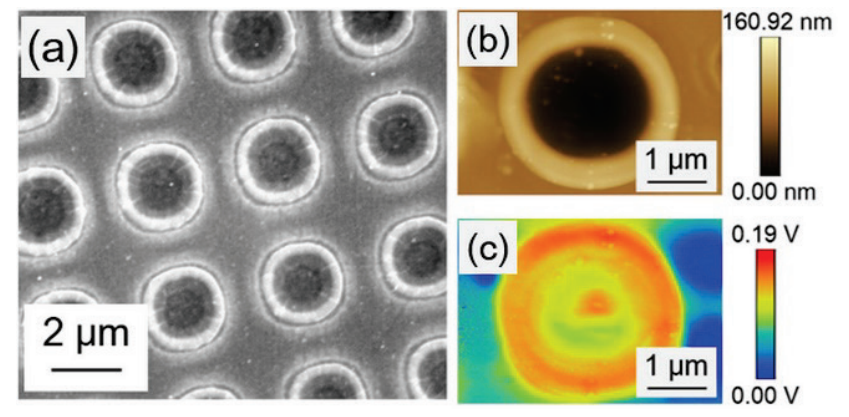

Fig. 5 (a) The SEM image of periodically micro-patterned $\mathrm{ZnO}$ buffer layer. (b) The AFM image of the ablated mark. (c) The KPFM image of the ablated mark.
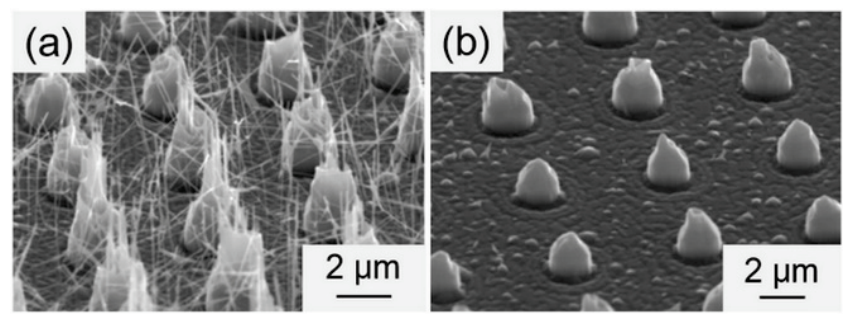

Fig. 6 (a) The SEM image of $\mathrm{ZnO}$ microcrystals and nanowires grown on the periodically micro-patterned $\mathrm{ZnO}$ buffer layer and (b) its SEM image after ultrasonic cleaning process.

$\mathrm{ZnO}$ microcrystals and nanowires were grown on the micro-patterned ZnO buffer layer by NAPLD. Same experimental conditions were employed as those of section 2.3. Figure 6(a) shows a $40^{\circ}$ tilted-view SEM image of asgrown $\mathrm{ZnO}$ nano/microcrystals. The surface of $\mathrm{ZnO}$ microcrystals were covered with many randomly-grown $\mathrm{ZnO}$ nanowires. These nanowires were removed by ultrasonic cleaning process, so that periodically-aligned $\mathrm{ZnO}$ microcrystals were remained, as shown in Fig. 6(b).

When the diameter of patterns is larger, $\mathrm{ZnO}$ microcrystals have hollow structures. [9] Therefore, we believe that $\mathrm{ZnO}$ microcrystals were formed by coalescing $\mathrm{ZnO}$ nanowires each other. These $\mathrm{ZnO}$ microcrystals with diameters of $1.5 \mu \mathrm{m}$ can be applied to fabricate field emitter arrays and $\mathrm{ZnO}$ microcrystals-based LED arrays without catalyst. Furthermore, the diameter and aspect ratio of periodically-aligned $\mathrm{ZnO}$ microcrystals can be controlled by adjusting laser parameters such as the fluence and pulse width as indicated in Fig. 4(b).

\section{Conclusion}

We found that the number density of $\mathrm{ZnO}$ nanowires is affected by pre-laser irradiation on $\mathrm{ZnO}$ buffer layers. Decreasing effect of $\mathrm{ZnO}$ nanowires was observed at pre-laser annealed $\mathrm{ZnO}$ buffer layer at $300 \mathrm{~mJ} / \mathrm{cm}^{2}$. Increasing effect of $\mathrm{ZnO}$ nanowires was determined at pre-laser ablated $\mathrm{ZnO}$ buffer layer at $500 \mathrm{~mJ} / \mathrm{cm}^{2}$. It was found that work functions were varied depending on laser fluences by the investigation about surface modification on $\mathrm{ZnO}$ buffer layer of laser irradiation by KPFM. Additionally, it was found that more densely-packed $\mathrm{ZnO}$ nanowires can be grown on the region with lower work function by NAPLD. KPFM can be a new intuitive method to evaluate locational surface properties of $\mathrm{ZnO}$ thin films. Finally, periodically-aligned $\mathrm{ZnO}$ microcrystals were fabricated on micro-patterned $\mathrm{ZnO}$ buffer layer using four-beam interfered laser. Since these techniques require only UV laser processing, they can develop the application of $\mathrm{ZnO}$ micro/nano crystals due to their high-throughput.

\section{Acknowledgments}

This work was supported by a Grant-in-Aid for Scientific Research from the Japan Society for the Promotion of Science (JSPS, No. 25286071).

\section{References}

[1] J. H. Lim, C. K. Kang, K. K. Kim, I. K. Park, D. K. Hwang, and S. J. Park: Adv. Mater., 18, (2006) 2720.

[2] S. Chu, J. H. Lim, L. J. Mandalapu, Z. Yang, L. Li, and J. L. Liu: Appl. Phys. Lett., 92, (2008) 152103.

[3] H. Zhang, J. Feng, J. Wang, and M. Zhang: Materials Letters, 61, (2007) 5202.

[4] Y. W. Heo, V. Varadarajan, M. Kaufman, K. Kim, and D. P. Norton: Appl. Phys. Lett., 81, 3046 (2002) 3048.

[5] Z. L. Wang: Materialstoday, 7, (2004) 26.

[6] T. Ohshima, R. K. Thareja, T. Ikegami, and K. Ebihara: Surface and Coating Technology, 169-170, (2003) 517.

[7] V. Craciun, J. Elders, J. G. E. Fardeniers, and I. W. Boyd: Appl. Phys. Lett., 65, (1994) 2963.

[8] R. Q. Guo, J. Nishimura, M. Matsumoto, D. Nakamura, and T. Okada: Appl. Phys. A, 93, (2008) 843.

[9] D. Nakamura, T. Shimogaki, S. Nakao, K. Harada, Y. Muraoka, H. Ikenoue, and T. Okada: J. Phys. D: Appl. Phys., 47, (2014) 034014.

[10] Y. Zhao, and Y. Jiang: J. Appl. Phys., 103, (2008) 114903.

[11] T. Shimogaki, K. Okazaki, D. Nakamura, M. Higashihata, T. Asano, and T. Okada: Opt. Express, 20, (2012) 15247.

[12] S. Kurbanov, W. C. Yang, and T. W. Kang: Appl. Phys. Express, 4, (2011) 021101.

[13]K. H. Tam, C. K. Cheung, Y. H. Leung, A. B. Djurišić, C. C. Ling, C. D. Beling, S. Fung, W. M. Kwok, W. K Chan, D. L. Phillips, L Ding, and W. K. Ge: J. Phys. Chem. B, 110, (2006) 20865.

[14] Y. Nakata, N. Miyanaga, and T. Okada: Applied surface Science, 253, (2007) 6555.

(Received: June 27, 2014, Accepted: May 20, 2015) 